\title{
On the saddle point property of Abresch-Langer curves under the curve shortening flow
}

\author{
Thomas KwoK-Keung Au
}

\begin{abstract}
In the study of the curve shortening flow on general closed curves in the plane, Abresch and Langer posed a conjecture that the homothetic curves can be regarded as saddle points between multi-folded circles and certain singular curves. In other words, these homothetic curves are the watershed between curves with a nonsingular future and those with singular future along the flow. In this article, we provide an affirmative proof to this conjecture.
\end{abstract}

\section{Introduction}

Let $\gamma_{0}$ be a given immersed closed plane curve. We consider the initial value problem of the curve shortening flow:

$$
\left\{\begin{array}{l}
\gamma_{t}(p, t)=-\kappa(p, t) \boldsymbol{\nu}(p, t), \\
\gamma(p, 0)=\gamma_{0}(p)
\end{array}\right.
$$

where $\gamma(p, t)$ is a family of curves with curvature $\kappa$ and unit (outward) normal $\boldsymbol{\nu}(p, t)$. The curve shortening flow for an embedded closed initial curve was completely characterized by the Grayson convexity theorem [11] and the Gage-Hamilton theorem [10]. The first asserts that the flow drives any such $\gamma_{0}$ to a convex curve whereas the second says that a convex curve shrinks to a round point. However, when $\gamma_{0}$ has self-intersections, it is easy to see that singularities may arise in the process. A typical example is the flow of the cardoid. The little loop of the cardoid contracts and develops a cusp when the large loop still exists. It is thus necessary to classify the singularity of the flow. A natural way of classification arises from the blow-up rate of the curvature into type I and type II singularities (Altschuler [2] and a series of important studies of Angenent, for examples,[6, 7]). In our situation, all the singularities are of type I, namely, $|\kappa|_{\max }(t) \sqrt{t_{\infty}-t} \leq C$, where $t_{\infty}$ is the blow-up time. In such a case, the singularity looks asymptotically like a contracting self-similar solution. 
A (contracting) self-similar solution of (0.1) is a flow in which the shapes of the curves change homothetically and continuously to a point in finite time. A curve in the flow is called a contracting self-similar curve. Obviously, the circle is a contracting self-similar curve. It turns out that other such curves must have self-intersections. In fact, all closed contracting self-similar solutions had been completely classified by Abresch and Langer,[1]. For our future discussion in this article, it is convenient to express their result in terms of the support function of the curve, since all these solutions are locally convex.

For each locally convex curve, $\gamma(p)$, the support function is a function

$$
h(\theta)=\langle\gamma(p), \boldsymbol{\nu}(p)\rangle,
$$

where $\theta$ is the angle of the outward normal and $\boldsymbol{\nu}=(\cos \theta, \sin \theta)$. The position of the curve $\gamma$ in terms of $h$ is given by

$$
\gamma(p)=\left(h(\theta) \cos \theta-h_{\theta}(\theta) \sin \theta, h(\theta) \sin \theta+h_{\theta}(\theta) \cos \theta\right) .
$$

This formulation is common in the discussions of convex plane curves. For reference, one may see [8]. With the expression of the curvature $\kappa$ in terms of $h$,

$$
\kappa(p)=\frac{1}{h(\theta)+h_{\theta \theta}(\theta)}
$$

Equation (0.1) can be rephrased as

$$
h_{t}(\theta, t)=\frac{-1}{h(\theta, t)+h_{\theta \theta}(\theta, t)}
$$

and the support function $h(\theta)$ of a contracting self-similar solution satisfies

$$
h_{\theta \theta}+h=\frac{C}{h}, \quad h>0, \quad C>0,
$$

where $C$ is a positive constant. Without loss of generality, from now on, we take $C=1$. Moreover, Equation (0.2) has a finite integral

$$
h_{\theta}^{2}+h^{2}=2 \log h+C_{0}
$$

It is easy to see that its solutions are positive and periodic. Let us denote by $h(\theta ; \alpha)$ the solution to $(0.2)$ satisfying the initial conditions

$$
h(0 ; \alpha)=\alpha>1 \quad \text { and } \quad h_{\theta}(0 ; \alpha)=0 .
$$


Then the main result in [1] can be stated as follows:

Abresch-Langer Theorem. First, the circle is the only embedded contracting self-similar curve. Second, as a increases from 1 to $\infty$, the period of $h(\cdot ; \alpha)$ decreases strictly from $2 \pi / \sqrt{2}$ to $\pi$.

Whenever the curve determined by the support function $h(\cdot ; \alpha)$ is closed, its period must be a multiple of $2 \pi$. In other words, for any pair of relatively prime positive integers $m, n$ satisfying $\frac{1}{2}<\frac{m}{n}<\frac{1}{\sqrt{2}}$, there corresponds a unique contracting self-similar curve with $n$ leaves in $m$ rotations (Figure 1). In this article, we follow the notation of Abresch-Langer to denote such a homothetic curve by $\gamma_{m, n}$ and, likewise, its support function by $h_{m, n}$.

Geometrically, the value $\alpha$ is the distance from the origin to the tip of a leave; and analytically, it is the maximum of $h, \alpha=\max _{\theta} h(\theta ; \alpha)$.

The linear stability properties of these contracting self-similar solutions are studied in [1] and Epstein-Weinstein [9]. As related to the nonlinear stability of these curves, Abresch and Langer explain that they serve in a certain sense as "saddle points" between nonsingular and singular curves. More precisely, their conjecture can be stated as follows.

Abresch-Langer Conjecture. Consider Equation (0.1) with a given initial data $\gamma_{0}=\gamma_{m, n}+\varepsilon \boldsymbol{\nu}$, with $|\varepsilon|$ small:

(a) when $\varepsilon>0$, the trajectory through $\gamma_{0}$ is asymptotic to an $m$-fold circle; and

(b) when $\varepsilon<0$, the trajectory through $\gamma_{0}$ is asymptotic to a singular curve $\Gamma_{m, n}$ with $n$ cusp points.
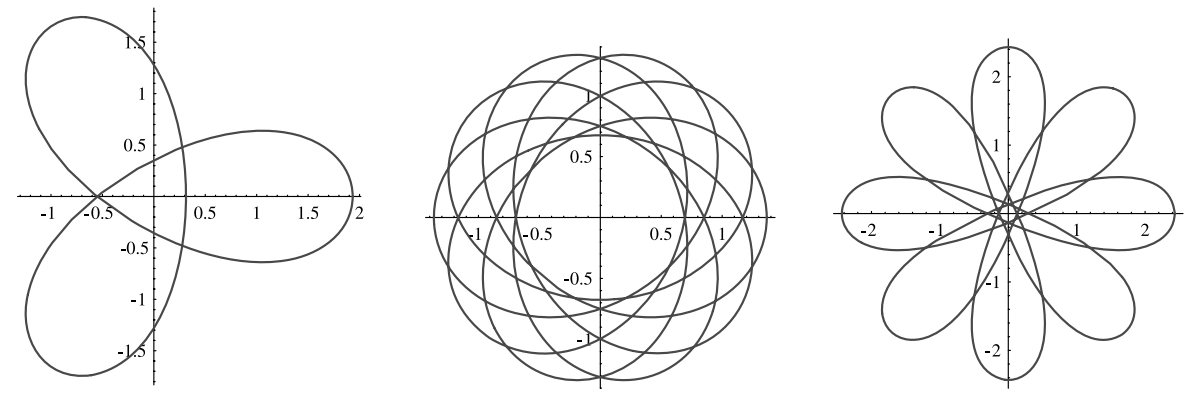

Figure 1: Pictures of $\gamma_{2,3}, \gamma_{7,10}$ and $\gamma_{5,8}$. 
They also expect the evolution process after rescaling behaves as shown in the pictures (Figure 2).

We shall prove the conjecture in this article. Furthermore, we also show analytically how the flow develops singularities in case (b) and the shape and structure of the curve is the same as the qualitative description in [1].

Main Theorem. The Abresch-Langer Conjecture is true.

(a) In the case that $\varepsilon>0$, solution to $(0.1)$ exists for all time $t \in[0, \infty)$ and it tends to an $m$-fold circle as $t \rightarrow \infty$.

(b) In the case that $\varepsilon<0$, $n$ singularity points are formed when the area of a leaf becomes zero and the curvature blows up.

Our discussion is organized in the following way. In Section 1, we formulate the problem in a suitable and convenient way. Several equivalent versions of the problem will be present; each of which will be used in later discussion. The formulation of the problem in terms of support function has been given in [8]. In fact, such formulation provides a clean and elegant view of the topic, which is essential to our solution of the problem. In Section 2, we establish bounds for the support function and the curvature to lay the foundation for applying maximum principle to the solution. It follows naturally with the derivative estimates and the convergence of the flow to self-similar solution in the case that $\varepsilon>0$. Then the instability of Abresch-Langer curves is shown. In Section 3, we deal with the case that $\varepsilon<0$. More specifically, we first show that the solution always exists before the second stage in figure $2 \mathrm{~b}$. It further exists as long as the leaves do not vanish. Since it is known that these leaves eventually shrink to a point, and at this moment, the singularities occur.

\section{Formulation and normalization}

First of all, let us reformulate the problem in terms of support functions of the curves. We would like to study the initial value problem of solving for $h(\theta, t)$,

$$
\left\{\begin{array}{l}
h_{t}(\theta, t)=\frac{-1}{h(\theta, t)+h_{\theta \theta}(\theta, t)}, \\
h(\theta, 0)=h_{m, n}(\theta)+\varepsilon
\end{array}\right.
$$


(a)
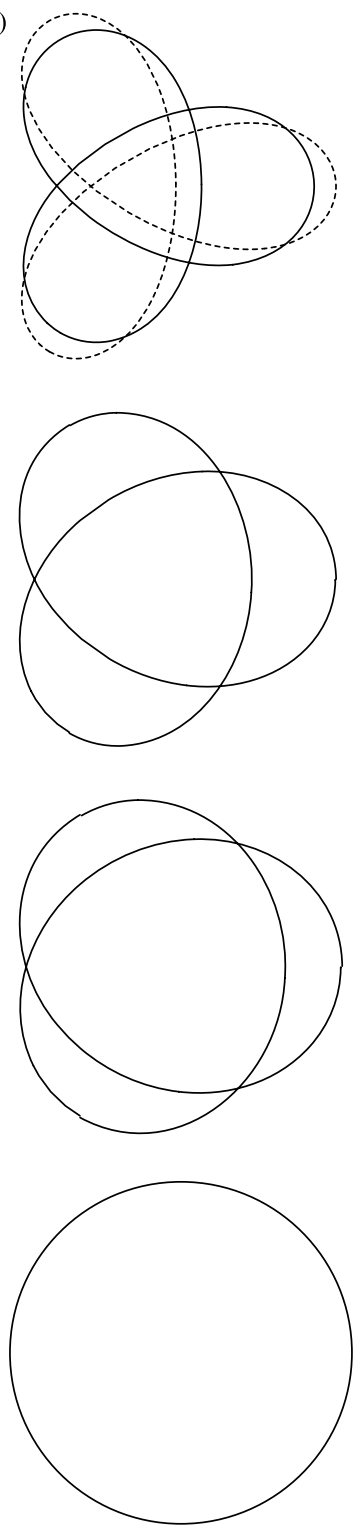

(b)
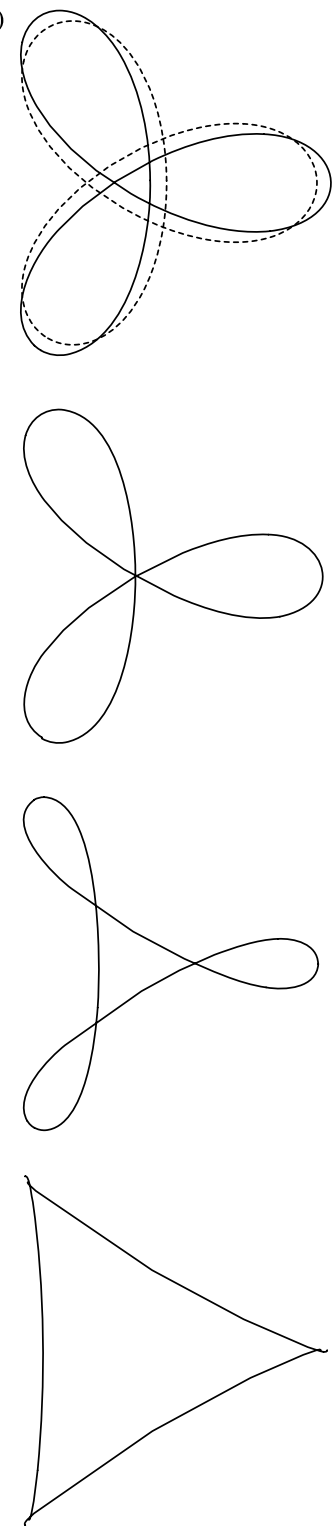

Figure 2: (a) Rescaled evolution for $\varepsilon>0$. (b) Rescaled evolution for $\varepsilon<0$. These pictures are numerically produced with a Mathematica algorithm which is mostly according to the flow with minor modification to overcome the slow convergence of the program. All the curves have been rescaled. The top pictures show homothetic curve $\gamma_{2,3}$ in dash and its perturbation $\gamma_{2,3}+\varepsilon \boldsymbol{\nu}$ in solid. 
In order to compare the problem with the situation at $\varepsilon=0$, we replace $h_{m, n}+\varepsilon$ with

$$
h_{\varepsilon}(\theta)=\left(1+\frac{L}{m \pi} \varepsilon+\varepsilon^{2}\right)^{-1 / 2}\left(h_{m, n}(\theta)+\varepsilon\right)
$$

where $L$ is the arc length of $\gamma_{m, n}$. Next, the algebraic area of the curve $\gamma$ can be determined by the support function $h$, namely,

$$
A(h)=\frac{1}{2} \int_{0}^{2 m \pi} h\left(h+h_{\theta \theta}\right) d \theta
$$

Then, $A\left(h_{m, n}\right)=A\left(h_{\varepsilon}\right)=m \pi$ with the above choice of initial support function. Let $h(\theta, t)$ be the periodic solution to the evolution equation for $\theta \in[0,2 m \pi]$ and $t \geq 0$,

$$
\left\{\begin{array}{l}
h_{t}(\theta, t)=\frac{-1}{h(\theta, t)+h_{\theta \theta}(\theta, t)} \\
h(\theta, 0)=h_{\varepsilon}(\theta)
\end{array}\right.
$$

We then have

$$
\begin{aligned}
\frac{\partial A(h(\cdot, t))}{\partial t} & =\frac{1}{2} \int_{0}^{2 m \pi} h_{t}\left(h+h_{\theta \theta}\right)+h\left(h_{t}+h_{t \theta \theta}\right) d \theta \\
& =\int_{0}^{2 m \pi} h_{t}\left(h+h_{\theta \theta}\right) \quad \text { after twice integrating by parts, } \\
& =\int_{0}^{2 m \pi}(-1)=-2 m \pi
\end{aligned}
$$

In other words, $A(h)=A_{0}-2 m \pi t=m \pi(1-2 t)$. We further normalize the flow by letting

$$
\tilde{h}(\theta, t)=\frac{1}{\sqrt{1-2 t}} h(\theta, t)
$$

and changing of variables from $t$ to $\tau$ such that $\frac{d \tau}{d t}=1 /(1-2 t)$. We have the following initial value problem for $\theta \in[0,2 m \pi]$ and $\tau \geq 0$ :

$$
\left\{\begin{array}{l}
\tilde{h}_{\tau}(\theta, \tau)=-\tilde{\kappa}(\theta, \tau)+\tilde{h}(\theta, \tau), \\
\tilde{h}(\theta, 0)=h_{\varepsilon}(\theta) .
\end{array}\right.
$$


As long as $A(h)>0,(1.4)$ is equivalent to (1.3). This is true in particular if $h>0$. Under this formulation, $h_{m, n}$ is a stationary solution to (1.4). Furthermore, algebraic area of the curve determined by $\tilde{h}(\cdot, \tau)$ is always equal to $m \pi$.

Let $\kappa_{\varepsilon}, \kappa$ and $\tilde{\kappa}$ denote the curvatures of the curves $\gamma_{\varepsilon}, \gamma$ and $\tilde{\gamma}$ with support functions $h_{\varepsilon}, h$ and $\tilde{h}$, respectively. Then they satisfy the following equations, which correspond to Equations (1.3) and (1.4), respectively:

$$
\begin{aligned}
\kappa_{t} & =\kappa^{2}\left(\kappa_{\theta \theta}+\kappa\right), \\
\tilde{\kappa}_{\tau} & =\tilde{\kappa}^{2}\left(\tilde{\kappa}+\tilde{\kappa}_{\theta \theta}\right)-\tilde{\kappa} .
\end{aligned}
$$

The details of the discussion in terms of support function can be referred to $[8]$.

\section{Outward perturbation}

In this section, we will deal with the case that $\varepsilon>0$. This corresponds to that the initial curve is a small outward perturbation of an Abresch-Langer self-similar curve.

Lemma 2.1. Let $\varepsilon>0$ and $h(\theta, t)$ be a $\frac{2 m \pi}{n}$-periodic solution to initial value problem (1.3). Then

$$
\max _{\theta} h(\theta, t)=h(0, t) ; \quad \min _{\theta} h(\theta, t)=h(m \pi / n, t)
$$

and, $h$ is decreasing for $\theta \in[0, m \pi / n]$. Furthermore, let $\kappa$ be the curvature of the curve supported by $h$, then $\max _{\theta} \kappa(\theta, t)=\kappa(0, t)$ and $\min _{\theta} \kappa(\theta, t)=$ $\kappa(m \pi / n, t)$.

Proof. Firstly, for all $k \geq 1, h_{\varepsilon}^{(k)}=\left(1+\frac{L}{m \pi} \varepsilon+\varepsilon^{2}\right)^{-1 / 2} h_{m, n}^{(k)}$. Therefore, $h_{\varepsilon}$ and $h_{m, n}$ have the same critical points with the same the extremal properties. As a result,

$$
\max _{\theta} h_{\varepsilon}(\theta)=h_{\varepsilon}(0), \quad \quad \min _{\theta} h_{\varepsilon}(\theta)=h_{\varepsilon}(m \pi / n)
$$

and $h_{\varepsilon}$ decreases for $\theta \in[0, m \pi / n]$. Secondly, from the evolution equation,

$$
h_{t \theta}=\frac{1}{\left(h+h_{\theta \theta}\right)^{2}}\left(h_{\theta}+h_{\theta \theta \theta}\right) \text {. }
$$


This shows that $h_{\theta}$ satisfies a parabolic equation, $\left(h_{\theta}\right)_{t}=\kappa^{2}\left(h_{\theta}\right)_{\theta \theta}+\kappa^{2}\left(h_{\theta}\right)$. By Sturm oscillation theorem [5], the number of zeros of $h_{\theta}$ is nonincreasing in $t$. Thus, for all $t>0, h$ has at most 2 critical points in $[0, m \pi / n]$.

On the other hand, by symmetry of $h$ along $\theta=0$ and $m \pi / n$, we must have $h_{\theta}=0$ at the symmetry. Hence for each $t, h(\theta, t)$ has exactly one maximum and one minimum for $\theta \in[0, m \pi / n]$. The desired results for $h$ follow easily.

The proof for the extremal of $\kappa$ is similar by simply observing that

$$
\kappa_{\varepsilon}=\left(1+\frac{L}{m \pi} \varepsilon+\varepsilon^{2}\right)^{1 / 2} \frac{\kappa_{m, n}}{1+\varepsilon \kappa_{m, n}} ;
$$

and the equation for $\kappa_{\theta}$ is $\left(\kappa_{\theta}\right)_{t}=\kappa^{2}\left(\kappa_{\theta}\right)_{\theta \theta}+2 \kappa \kappa_{\theta}\left(\kappa_{\theta}\right)_{\theta}+3 \kappa^{2} \kappa_{\theta}$.

\section{Remark.}

- From the above proof, we indeed have some information about the shape of each leaf of the immersed curve defined by such $h(\theta, t)$. In particular, both the longest distance (the tip of a leaf) and the shortest distance from the origin to the curve are attained along the same directions as those of $\gamma_{m, n}$.

- The same statement as the above lemma is valid for the functions $\tilde{h}$ and $\tilde{\kappa}$.

- Besides being $\frac{2 m \pi}{n}$-periodic, all the functions $h, \kappa, \tilde{h}, \tilde{\kappa}$ are symmetric about $\frac{m \pi}{n}$, i.e., $h(\theta, t)=h(m \pi / n-\theta, t)$, etc.

Lemma 2.2. For sufficiently small $\varepsilon>0$, we have

$$
h_{\varepsilon}(0)<h_{m, n}(0) \quad \text { and } \quad h_{\varepsilon}(m \pi / n)>h_{m, n}(m \pi / n) .
$$

Moreover, $\kappa_{\varepsilon}(0)<\kappa_{m, n}(0)$ and $\kappa_{\varepsilon}(m \pi / n)>\kappa_{m, n}(m \pi / n)$. All these inequalities reverse for $\varepsilon<0$.

Proof. By definition of $h_{\varepsilon}$ and its expansion in $\varepsilon$, we have

$$
h_{\varepsilon}=h_{m, n}+\left(1-\frac{L}{2 m \pi} h_{m, n}\right) \varepsilon+O\left(\varepsilon^{2}\right) .
$$

Note that $h_{m, n}=\kappa_{m, n}$, so

$$
\int_{0}^{2 m \pi} \frac{1}{h_{m, n}}=\int_{0}^{2 m \pi} \frac{1}{\kappa_{m, n}}=\int_{0}^{2 m \pi}\left[h_{m, n}+\left(h_{m, n}\right)_{\theta \theta}\right]=\int_{0}^{2 m \pi} h_{m, n}=L .
$$


Applying the Mean Value Theorem, there is a $\theta_{*}$ such that $\frac{1}{h_{m, n}\left(\theta_{*}\right)}(2 m \pi)=L$. According to the preceding Lemma 2.1, $h_{m, n}(0)>h_{m, n}\left(\theta_{*}\right)^{>}>h_{m, n}(m \pi / n)$. As a consequence,

$$
1-\frac{L}{2 m \pi} h_{m, n}(0)<0, \quad 1-\frac{L}{2 m \pi} h_{m, n}(m \pi / n)>0 .
$$

The inequalities can be obtained by putting the above into the expansion of $h_{\varepsilon}$.

The proof for the inequalities of $\kappa$ is similar by observing that

$$
\kappa_{\varepsilon}=\kappa_{m, n}+\varepsilon \kappa_{m, n}\left(\frac{L}{2 m \pi}-\kappa_{m, n}\right)+O\left(\varepsilon^{2}\right),
$$

and applying the Mean Value Theorem to $\int_{0}^{2 m \pi} \kappa_{m, n}=\int_{0}^{2 m \pi} h_{m, n}=L$ to obtain the inequalities $\kappa_{m, n}(m \pi / n)<\frac{L}{2 m \pi}<\kappa_{m, n}(0)$.

In order to prove the long time existence of solutions, we need uniform bounds.

Proposition 2.1. For sufficiently small $\varepsilon>0$, the solutions $\tilde{h}$ to (1.4) and $\tilde{\kappa}$ to $\left(1.4^{*}\right)$ satisfy the following for all $\tau \geq 0$ :

$$
\begin{aligned}
& h_{m, n}\left(\frac{m \pi}{n}\right) \leq \tilde{h}(\theta, \tau) \leq h_{m, n}(0) \\
& \kappa_{m, n}\left(\frac{m \pi}{n}\right) \leq \tilde{\kappa}(\theta, \tau) \leq \kappa_{m, n}(0)
\end{aligned}
$$

This proposition will be established through a number of steps. We will first state the following two lemmas, corresponding to the bounds for $\tilde{h}$ and $\tilde{\kappa}$, respectively.

Lemma 2.3. For sufficiently small $\varepsilon>0$, for any $\tau \geq 0$, there are exactly two (counting multiplicity) $\theta_{1} \in\left[0, \frac{m \pi}{n}\right]$ and $\theta_{2}=\frac{2 m \pi}{n}-\theta_{1} \in\left[\frac{m \pi}{n}, \frac{2 m \pi}{n}\right]$ such that $h_{m, n}\left(\theta_{j}\right)=\tilde{h}\left(\theta_{j}, \tau\right), j=1,2$.

Lemma 2.4. For sufficiently small $\varepsilon>0$, for any $\tau \geq 0$, there are exactly two (counting multiplicity) $\theta_{1} \in\left[0, \frac{m \pi}{n}\right]$ and $\theta_{2}=\frac{2 m \pi}{n}-\theta_{1} \in\left[\frac{m \pi}{n}, \frac{2 m \pi}{n}\right]$ such that $\kappa_{m, n}\left(\theta_{j}\right)=\tilde{\kappa}\left(\theta_{j}, \tau\right), j=1,2$. 
Proof of Proposition 2.1. Let us first complete the proof assuming Lemmas 2.3 and 2.4. From Lemmas 2.1 and 2.2, all the functions $\tilde{h}(\cdot, \tau), h_{\varepsilon}, h_{m, n}$ have maxima at $\theta=0$ and minima at $\theta=m \pi / n$. Also, from Lemma 2.3, $\tilde{h}(\cdot, \tau)-h_{m, n}$ has the number of zeros unchanged as its initial function $h_{\varepsilon}-h_{m, n}$, which has $h_{\varepsilon}(0)-h_{m, n}(0)<0$ and $h_{\varepsilon}(m \pi / n)-h_{m, n}(m \pi / n)>0$. It follows that $\tilde{h}(\cdot, \tau)-h_{m, n}$ has the analogous inequalities. As a result

$$
\begin{aligned}
\max _{\theta} \tilde{h}(\theta, \tau)<\max _{\theta} h_{m, n} & =h_{m, n}(0), \\
\min _{\theta} \tilde{h}(\theta, \tau)>\min _{\theta} h_{m, n} & =h_{m, n}(m \pi / n) .
\end{aligned}
$$

The situation for $\tilde{\kappa}(\cdot, \tau)-\kappa_{m, n}$ is exactly the same following Lemma 2.4.

Proof of Lemma 2.3. We will start with showing for sufficiently small $\varepsilon>0$, there are exactly two simple zeros of $h_{\varepsilon}-h_{m, n}$, namely, $\theta_{1}$ and $\theta_{2}=\frac{2 m \pi}{n}-$ $\theta_{1} \in\left[0, \frac{2 m \pi}{n}\right]$ such that $h_{m, n}\left(\theta_{j}\right)=h_{\varepsilon}\left(\theta_{j}\right), j=1,2$.

Consider the initial function and its derivation wrt $\theta$,

$$
\begin{aligned}
& h_{\varepsilon}(\theta)=\left(1+\frac{L}{m \pi} \varepsilon+\varepsilon^{2}\right)^{-1 / 2}\left(h_{m, n}(\theta)+\varepsilon\right), \\
& h_{\varepsilon}^{\prime}(\theta)=\left(1+\frac{L}{m \pi} \varepsilon+\varepsilon^{2}\right)^{-1 / 2} h_{m, n}^{\prime}(\theta) .
\end{aligned}
$$

Let $g: \mathbb{R} \rightarrow \mathbb{R}$ be the function

$$
g(\theta): \stackrel{\operatorname{def}}{=}\left(\left.\frac{\partial h_{\varepsilon}}{\partial \varepsilon}\right|_{\varepsilon=0}\right)(\theta) .
$$

By a direct computation, we have

$$
\begin{aligned}
g(\theta) & =1-\frac{L}{2 m \pi} h_{m, n}(\theta) \\
g^{\prime}(\theta) & =\frac{-L}{2 m \pi} h_{m, n}^{\prime}(\theta)=\left.\frac{\partial}{\partial \varepsilon}\left(\frac{\partial h_{\varepsilon}}{\partial \theta}\right)\right|_{\varepsilon=0}
\end{aligned}
$$

From Lemma 2.2, we have $g(0)<0$ and $g\left(\frac{m \pi}{n}\right)>0$. Thus, there exists $\theta_{1} \in\left(0, \frac{m \pi}{n}\right)$ such that $g\left(\theta_{1}\right)=0$. Since $g$ also has the same symmetry of $h_{\varepsilon}$, hence $g\left(\theta_{2}\right)=0$ where $\theta_{2}=\frac{2 m \pi}{n}-\theta_{1}$. For all $\theta \in\left(0, \frac{m \pi}{n}\right) \cup\left(\frac{m \pi}{n}, \frac{2 m \pi}{n}\right)$, $g^{\prime}(\theta)=\frac{-L}{2 m \pi} h_{m, n}^{\prime}(\theta) \neq 0$. This is true in particular for $\theta_{1}$ and $\theta_{2}$. Thus, the zeros of $g$ at $\theta_{1}$ and $\theta_{2}$ are simple and they are the only ones in $\left[0, \frac{2 m \pi}{n}\right]$. By continuity, the same conclusion holds for $\tilde{h}(\cdot, \tau)-h_{m, n}$ for small $\varepsilon>0$.

We will then continue to prove the statement of the lemma. 
As $\tilde{h}(\cdot, \tau)-h_{m, n}$ satisfies a parabolic equation with initial function $h_{\varepsilon}-$ $h_{m, n}$, there are two cases concerning the number of zeros. Either, in the first case, the number of zeros remains constant for all $\tau \geq 0$; or, in the second case, it remains constant for $0 \leq \tau<\tau_{0}$, until a double zero is developed at $\tilde{h}\left(\cdot, \tau_{0}\right)-h_{m, n}$ and afterwards, $\tilde{h}(\cdot, \tau)-h_{m, n} \neq 0$ for all $\tau>\tau_{0}[4,5]$. We will show that the latter case does not occur.

Let us assume that after the double zero is developed, $\tilde{h}\left(\cdot, \tau_{1}\right)<h_{m, n}$ for $\tau_{1}>\tau_{0}$ (the other inequality can be handled similarly). Denote the curves determined by the support functions $\tilde{h}\left(\cdot, \tau_{1}\right)$ and $h_{m, n}$, respectively, by $\tilde{\gamma}$ and $\gamma_{m, n}$. Assume $(\tilde{r}(\phi), \phi)$ and $(r(\phi), \phi)$ are the parametrizations by polar coordinates of these two curves. We claim that the above inequality between $\tilde{h}$ and $h_{m, n}$ will force that $\tilde{r}<r$. This leads to a contradiction with the fact that both curves have the same algebraic area.

To show that $\tilde{r}<r$, suppose there is a polar angle $\phi_{0}$ such that $\tilde{r}\left(\phi_{0}\right)=$ $r\left(\phi_{0}\right)$. As the curves are locally convex with $\tilde{h}\left(\cdot, \tau_{1}\right)<h_{m, n}$, the intersection of $\tilde{\gamma}$ and $\gamma_{m, n}$ is transversal. Without loss of generality, assume that there is $\delta>0$ such that

$$
\tilde{r}(\phi)<r(\phi), \phi \in\left(\phi_{0}-\delta, \phi_{0}\right) \quad \text { and } \quad \tilde{r}(\phi)>r(\phi), \phi \in\left(\phi_{0}, \phi_{0}+\delta\right) .
$$

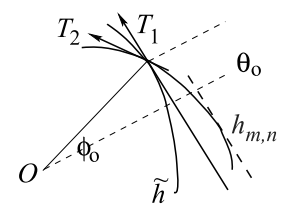

Let $T_{1}$ be the tangent to $\tilde{\gamma}$ at the transversal intersection, where the normal is $\left(\cos \theta_{0}, \sin \theta_{0}\right)$. Also, let $\mathbb{R}^{2} \backslash T_{1}=H_{1} \cup H_{2}$ where $H_{j}$ 's are half-planes with $H_{1}$ containing the origin $(0,0)$. Then $\left.\gamma_{m, n}\right|_{\left(\phi_{0}-\delta, \phi_{0}\right)} \subset H_{2}$, and $\left.\gamma_{m, n}\right|_{\left(\phi_{0}, \phi_{0}+\delta\right)}$ $\subset H_{1}$. In such a case, one must have $h_{m, n}\left(\theta_{0}\right)>\widetilde{h}\left(\theta_{0}, \tau_{0}\right)$. This violates the assumption above.

Consequently, after the double zero is developed, either $\tilde{r} \geq r$ or $\tilde{r} \leq r$. Thus, $A(\tilde{h})>A\left(h_{m, n}\right)$ or $A(\tilde{h})<A\left(h_{m, n}\right)$, which contradicts the normalization of algebraic area. This shows that only the first case is possible, i.e., for all $\tau \geq 0, \tilde{h}(\cdot, \tau)-h_{m, n}(\cdot)$ has exactly two zeros, both are simple, in $\left[0, \frac{2 m \pi}{n}\right]$.

Proof of Lemma 2.4. The proof involves establishing uniform bounds for the curvature function.

Starting from the uniform upper and positive lower bounds for $\tilde{h}$, we will deduce two-sided bounds on the curvature of $\tilde{\gamma}$ by expressing the curve 
as a local graph. By the uniform bounds of $\tilde{h}$, the ball centered at the origin and with radius $R=h_{m, n}(m \pi / n)$ is always contained inside the curve $\tilde{\gamma}$ corresponding to the support function $\tilde{h}$.

By choosing a suitable coordinates, the portion of the curve $\tilde{\gamma}$ corresponding to $\tilde{h}(\theta, \tau), \theta \in[-\delta, \delta]$, is a graph $\tilde{u}(x, \tau)$ over the interval $[-R, R]$. More precisely, we may express $\tilde{\gamma}(\theta(x), \tau)=(x, \tilde{u}(x, \tau)), x \in[-R, R]$. Then, under this local parametrization and the fact that, $\tilde{\gamma}_{\tau}=\tilde{\kappa} \boldsymbol{\nu}+\tilde{\gamma}$, we have the following computations:

$$
\begin{aligned}
\tilde{\gamma}_{\tau} & =\frac{\partial x}{\partial \tau}\left(1, \tilde{u}_{x}\right)+\left(0, \tilde{u}_{\tau}\right) \\
\boldsymbol{\nu} & =\frac{1}{\sqrt{1+\tilde{u}_{x}^{2}}}\left(-\tilde{u}_{x}, 1\right), \\
\tilde{\kappa} & =\frac{\tilde{u}_{x x}}{\left(1+\tilde{u}_{x}^{2}\right)^{3 / 2}}
\end{aligned}
$$

Moreover, Equation (1.4) becomes

$$
\tilde{u}_{\tau}=\frac{\tilde{u}_{x x}}{1+\tilde{u}_{x}^{2}}-x \tilde{u}_{x}+\tilde{u}
$$

In addition, $\tilde{u}$ is a concave function and so we have the estimate

$$
\sup \left\{\left|\tilde{u}_{x}\right|: x \in\left[\frac{-R}{2}, \frac{R}{2}\right]\right\} \leq \frac{2}{R} \operatorname{osc}\{\tilde{u}(x, \tau): x \in[-R, R]\} \leq \frac{2}{R} h_{m, n}(0)
$$

That is, $\tilde{u}_{x}$ is uniformly bounded over $\left[\frac{-R}{2}, \frac{R}{2}\right]$. So, $\tilde{u}$ satisfies a linear parabolic equation with bounded coefficients in $[-R, R] \times[0, \infty)$. By parabolic regularity [13], we obtain $C^{k}$-bounds on $\tilde{u}$ for all $k$. In particular, the curvature $\tilde{\kappa}=\frac{\tilde{u}_{x x}}{\left(1+\tilde{u}_{x}\right)^{3 / 2}}$ of $\tilde{\gamma}$, at the maximum, is bounded from above.

To obtain a positive lower bound for the curvature, it suffices to represent the curve $\tilde{\gamma}$ as a local graph in the neighborhood of the minimum at $\theta=m \pi / n$. The the graph $(x, \tilde{u})$ also satisfies (3.6) above. Using Harnack's Inequality, we obtain a positive lower bound for the curvature in terms of its maximum bound [13].

After establishing both side estimates, we can appeal again to parabolic regularity to Equation (1.4) and obtain uniform bounds of all orders. 
Combining Lemma 2.2 and Proposition 2.1, for sufficiently small $\varepsilon>0$, the curvature $\tilde{\kappa}(\theta, \tau)$ has uniformly bounds on both sides for all $\theta$ and for all $\tau \geq 0$. Thus, applying the regularity theory $[12,13,15]$ of uniformly parabolic equations to the linearized equation of Equation (1.4), one may infer that bounds for the solution exist for all orders. Hence, the solution exists globally.

Proposition 2.2. For any sequence $\tau_{j} \rightarrow \infty$, there is a subsequence $\tau_{j_{k}} \rightarrow \infty$ such that the corresponding sequence $\tilde{h}\left(\cdot, \tau_{j_{k}}\right)$ of solutions to Equation (1.4) converges to $h_{\infty}$ with $h_{\infty}=\kappa_{\infty}$, that is, either $h_{\infty}=h_{m, n}$ or it defines the $m$-fold circle.

Proof. We consider the entropy $\mathcal{F}(\tau)=\int_{0}^{2 m \pi} \log \tilde{h}(\theta, \tau) d \theta$ for the normalized flow $\left(1.4^{*}\right)$. Then

$$
\begin{aligned}
\mathcal{F}^{\prime}(\tau) & =\int_{0}^{2 m \pi} \frac{1}{\tilde{h}}(-\tilde{\kappa}+\tilde{h}) d \theta=-\int_{0}^{2 m \pi} \frac{\tilde{\kappa}}{\tilde{h}} d \theta+2 m \pi \\
& \leq-\left(\int_{0}^{2 m \pi} \frac{\tilde{h}}{\tilde{\kappa}} d \theta\right)^{-1}(2 m \pi)^{2}+2 m \pi \leq-\frac{4 m^{2} \pi^{2}}{2 m \pi}+2 m \pi=0 .
\end{aligned}
$$

Note that in the above,

$$
\left(\int_{0}^{2 m \pi} \frac{\tilde{\kappa}}{\tilde{h}} d \theta\right)\left(\int_{0}^{2 m \pi} \frac{\tilde{h}}{\tilde{\kappa}} d \theta\right) \geq\left(\int_{0}^{2 m \pi} 1\right)^{2}
$$

and equality holds if and only if $\tilde{\kappa} / \tilde{h}$ is a constant.

It follows that

$$
\mathcal{F}(\tau)-\mathcal{F}(0)=\int_{0}^{\tau}\left(\int_{0}^{2 m \pi} \frac{-\tilde{\kappa}}{\tilde{h}} d \theta+2 m \pi\right) d \tau .
$$

Since both $\mathcal{F}(\tau)$ and $\mathcal{F}(0)$ are of finite values and bounded for all $\tau \geq 0$, there exists constant $C_{1}$ such that

$$
\int_{0}^{\infty}\left(\int_{0}^{2 m \pi} \frac{-\tilde{\kappa}}{\tilde{h}} d \theta+2 m \pi\right) d \tau \leq C_{1}<\infty
$$

Let $\tau_{j}$ be a sequence with $\tau_{j} \rightarrow \infty$, we claim that $\inf _{j} \mathcal{F}^{\prime}\left(\tau_{j}\right)=0$. Otherwise, there exists $\eta>0$ such that $\left|\mathcal{F}^{\prime}\left(\tau_{j}\right)\right| \geq \eta>0$. By uniform estimate, there 
exists $\delta>0$, independent of $\tau_{j}$ with $\left|\mathcal{F}^{\prime}\left(\tau_{j}\right)\right| \geq \eta / 2$ on $\left[\tau_{j}, \tau_{j}+\delta\right]$ and hence one has the contradiction that

$$
\int_{0}^{\infty}\left|\mathcal{F}^{\prime}(\tau)\right| d \tau=\infty
$$

Now, pick a subsequence $\tau_{j_{k}} \rightarrow \infty$ that $\mathcal{F}^{\prime}\left(\tau_{j_{k}}\right) \rightarrow 0$, that is,

$$
\int_{0}^{2 m \pi} \frac{\tilde{\kappa}\left(\theta, \tau_{j_{k}}\right)}{\tilde{h}\left(\theta, \tau_{j_{k}}\right)} d \theta \rightarrow 2 m \pi
$$

Since $\tilde{h}$ already has $C^{2, \alpha}$-bound, passing to the subsequence,

$$
\tilde{h}\left(\cdot, \tau_{j_{k}}\right) \rightarrow h_{\infty}, \quad \text { which has } \int_{0}^{2 m \pi} \frac{\kappa_{\infty}}{h_{\infty}} d \theta=2 m \pi .
$$

By Hölder's property and the normalization of constant area, $h_{\infty}=\kappa_{\infty}$. Thus, either $h_{\infty}=h_{m, n}$ or it determines the $m$-fold circle.

We will rule out that it converges back to an Abresch-Langer curve. To prepare for this, we first write down further expansions of $h_{\varepsilon}$ and its corresponding curvature $\kappa_{\varepsilon}$ :

$$
\begin{aligned}
h_{\varepsilon}= & h_{m, n}+\varepsilon\left(1-\frac{L}{2 m \pi} h_{m, n}\right)+\varepsilon^{2}\left(\frac{-1}{2} h_{m, n}-\frac{L}{2 m \pi}+\frac{3 L^{2}}{8 m^{2} \pi^{2}} h_{m, n}\right) \\
& +O\left(\varepsilon^{3}\right), \\
\kappa_{\varepsilon}= & \kappa_{m, n}\left[1+\varepsilon\left(\frac{L}{2 m \pi}-\kappa_{m, n}\right)+\varepsilon^{2}\left(\frac{1}{2}+\kappa_{m, n}^{2}-\frac{3 L^{2}}{8 m^{2} \pi^{2}}-\frac{L}{2 m \pi} \kappa_{m, n}\right)\right] \\
& +O\left(\varepsilon^{3}\right) .
\end{aligned}
$$

Consider the entropy $\mathcal{E}(\varepsilon)=\int_{0}^{2 m \pi} \log \kappa_{\varepsilon} d \theta$. Then

$$
\begin{aligned}
\mathcal{E}^{\prime}(\varepsilon)= & \int_{0}^{2 m \pi} \frac{\kappa_{m, n}}{\kappa_{\varepsilon}}\left[\frac{L}{2 m \pi}-\kappa_{m, n}+\varepsilon\left(1+2 \kappa_{m, n}^{2}-\frac{L^{2}}{4 m^{2} \pi^{2}}\right.\right. \\
& \left.\left.-\frac{L \kappa_{m, n}}{m \pi}\right)+O\left(\varepsilon^{2}\right)\right], \\
\mathcal{E}^{\prime \prime}(\varepsilon)= & \int_{0}^{2 m \pi} \frac{\kappa_{m, n}}{\kappa_{\varepsilon}}\left(1+2 \kappa_{m, n}^{2}-\frac{L^{2}}{4 m^{2} \pi^{2}}-\frac{L \kappa_{m, n}}{m \pi}\right) \\
& -\frac{\kappa_{m, n}^{2}}{\kappa_{\varepsilon}^{2}}\left(\frac{L}{2 m \pi}-\kappa_{m, n}\right)^{2}+O(\varepsilon) .
\end{aligned}
$$


It follows that, using $\kappa_{m, n}=h_{m, n}$,

$$
\begin{aligned}
\mathcal{E}^{\prime \prime}(\varepsilon) & =2 m \pi-\frac{L^{2}}{m \pi}+\int_{0}^{2 m \pi} \kappa_{m, n}^{2} \\
& =2 m \pi\left[1-2\left(f h_{m, n}\right)^{2}+f h_{m, n}^{2}\right]+O(\varepsilon) .
\end{aligned}
$$

Similarly for $\mathcal{F}$, we may also work out the same calculations.

$$
\begin{aligned}
\mathcal{F}^{\prime}(\varepsilon)= & \int_{0}^{2 m \pi} \frac{1}{h_{m, n}}\left[1-\frac{L}{2 m \pi} h_{m, n}+\varepsilon\left(-h_{m, n}-\frac{L}{m \pi}\right.\right. \\
& \left.\left.+\frac{3 L^{2}}{4 m^{2} \pi^{2}} h_{m, n}\right)+O\left(\varepsilon^{2}\right)\right] \\
\mathcal{F}^{\prime \prime}(\varepsilon)= & \int_{0}^{2 m \pi} \frac{1}{h_{m, n}}\left(-h_{m, n}-\frac{L}{m \pi}+\frac{3 L^{2}}{4 m^{2} \pi^{2}} h_{m, n}\right) \\
& -\frac{1}{h_{m, n}^{2}}\left(1-\frac{L}{2 m \pi} h_{m, n}\right)^{2}+O(\varepsilon) .
\end{aligned}
$$

Using the fact that $1 / h_{m, n}=1 / \kappa_{m, n}=h_{m, n}+\left(h_{m, n}\right)_{\theta \theta}$, we have

$$
\mathcal{F}^{\prime \prime}(\varepsilon)=-2 m \pi\left[1-2\left(f \frac{1}{h_{m, n}}\right)^{2}+f \frac{1}{h_{m, n}^{2}}\right]+O(\varepsilon) .
$$

With these calculations, we have the following nonlinear instability result.

Proposition 2.3. $(\mathcal{E}+\mathcal{F})^{\prime}(0)=0$ and $(\mathcal{E}+\mathcal{F})^{\prime \prime}(0)<0$.

Proof. It is evident that $(\mathcal{E}+\mathcal{F})^{\prime}(0)=0$. Also, we have

$$
(\mathcal{E}+\mathcal{F})^{\prime \prime}(0)=2 m \pi\left[f h_{m, n}^{2}-\int \frac{1}{h_{m, n}^{2}}\right]
$$


Here, the second term becomes

$$
\begin{aligned}
\int \frac{1}{h_{m, n}^{2}} & =f\left[h_{m, n}+\left(h_{m, n}\right)_{\theta \theta}\right]^{2} \\
& =f h_{m, n}^{2}+2 f h_{m, n}\left(h_{m, n}\right)_{\theta \theta}+f\left(h_{m, n}\right)_{\theta \theta}^{2} \\
& =f h_{m, n}^{2}-2 f\left(h_{m, n}\right)_{\theta}^{2}+f\left(h_{m, n}\right)_{\theta \theta}^{2}
\end{aligned}
$$

By Poincaré's Inequality, we have $f\left(h_{m, n}\right)_{\theta \theta}^{2} \geq\left(\frac{2 \pi}{2 m \pi / n}\right)^{2} f\left(h_{m, n}\right)_{\theta}^{2}$.

Combining the above, together with $\frac{m}{n}<\frac{1}{\sqrt{2}}$, we have

$$
(\mathcal{E}+\mathcal{F})^{\prime \prime}(0)=\left[2-\frac{n^{2}}{m^{2}}\right] f\left(h_{m, n}\right)_{\theta}^{2}<0 .
$$

Now, we can finish the proof of part (a) in the Main Theorem. We have already known that the flow $\tilde{\gamma}$ exists for all $\tau \geq 0$. By Proposition 2.2, it converges either to the $m$-circle or back to its initial curve. As $\mathcal{E}+\mathcal{F}$ is nonincreasing along the flow, it follows from Proposition 2.3 that for sufficiently small $\varepsilon>0$ and for all $\tau \geq 0$,

$$
(\mathcal{E}+\mathcal{F})(\tilde{h}(\cdot, \tau)) \leq(\mathcal{E}+\mathcal{F})\left(h_{\varepsilon}\right)<(\mathcal{E}+\mathcal{F})\left(h_{m, n}\right)
$$

So, it must converge to the $m$-circle and (a) holds. Note that we work on Equation (1.4) which is obtained from normalization by the algebraic area. It is equivalent to the flow with normalization by arc length.

\section{Inward perturbation}

In this section, we will show that, for $\varepsilon<0$, the singularities occur exactly when the area of a leaf vanishes and the curvature of the curve blows up. We first establish some analytical results. The following lemma basically lays the foundation to show that in the evolution, the leaves eventually "shrink" and exclude the origin.

Lemma 3.1. Let $\tilde{h}(\theta, \tau)$ be a solution to the initial value problem (1.4). If $\tilde{h}>0$ for all $\tau$ in an interval, then $\tilde{h}$ is also uniformly bounded above. 
Proof. Modifying an argument in [10], we consider the following quantity, $W(\tau)$ :

$$
\begin{aligned}
W(\tau) & =\int_{-\pi / 2}^{\pi / 2} \frac{\cos \theta}{\tilde{\kappa}(\theta, \tau)}=\int_{-\pi / 2}^{\pi / 2}\left(\tilde{h}+\tilde{h}_{\theta \theta}\right) \cos \theta \\
& =\int_{-\pi / 2}^{\pi / 2} \tilde{h} \cos \theta+\left.\tilde{h}_{\theta} \cos \theta\right|_{-\pi / 2} ^{\pi / 2}+\left.\tilde{h} \sin \theta\right|_{-\pi / 2} ^{\pi / 2}-\int_{-\pi / 2}^{\pi / 2} \tilde{h} \cos \theta \\
& =\tilde{h}\left(\frac{\pi}{2}, \tau\right)+\tilde{h}\left(\frac{-\pi}{2}, \tau\right) .
\end{aligned}
$$

Therefore, $W(\tau)$ is the width (measured perpendicular to the longest axis) of a leaf of the curve defined by $\tilde{h}$. By Jensen's Inequality,

$$
\begin{aligned}
\log W(\tau) & \geq \int_{-\pi / 2}^{\pi / 2} \log \left(\frac{\cos \theta}{\tilde{\kappa}(\theta, \tau)}\right) \\
& =\int_{-\pi / 2}^{\pi / 2} \log \cos \theta-\int_{-\pi / 2}^{\pi / 2} \log \tilde{\kappa} \\
& =\log C-\mathcal{E}(\tau) \geq \log C-\mathcal{E}(0),
\end{aligned}
$$

for some constant $C>0$, because $\mathcal{E}$ is decreasing (proved in Proposition 2.2). Thus, $W(\tau)$ is uniformly bounded below. Since

$$
W(\tau) \cdot \max _{\theta} \tilde{h}(\theta, \tau) \leq \text { the area of a leaf } \leq A(h)=m \pi,
$$

it follows that $\max _{\theta} \tilde{h}(\theta, \tau)$ has a uniform upper bound.

Now, we may use this lemma to show that for $\varepsilon<0$, at some time, a curve in the flow will pass through the origin.

Proposition 3.1. Let $\tilde{h}(\theta, \tau)$ be the solution to the initial value problem (1.4) with $\varepsilon<0$. Then there is a time $\tau_{1}$ such that $\min _{\theta} \tilde{h}\left(\theta, \tau_{1}\right)=0$ and $\tilde{h}>0$ for $\tau \in\left[0, \tau_{1}\right)$.

Proof. Suppose on the contrary that $\tilde{h}>0$ for all $\tau \geq 0$. Thus the curve $\tilde{\gamma}$ at each $\tau$ contains uniformly a small ball inside. Just as in the proof of Lemma 2.4, one may locally express the tip of a leaf as a concave graph. 
Specifically, we write

$$
\tilde{\gamma}(\theta(x), \tau)=(x, \tilde{u}(x, \tau)), \quad x \in(-\ell, \ell),
$$

such that $\theta(0)=0$. By assumption and the preceding Lemma 3.1, there is $M>0$ such that $\max _{x} \tilde{u}(x, \tau) \leq M$ for all $\tau \geq 0$. Exactly as given in Lemma 2.4, we have

$$
\begin{aligned}
\tilde{\gamma}_{\tau} & =\tilde{\kappa} \boldsymbol{\nu}+\tilde{\gamma} \\
\tilde{\gamma}_{\tau} & =\frac{\partial x}{\partial \tau}\left(1, \tilde{u}_{x}\right)+\left(0, \tilde{u}_{\tau}\right), \\
\boldsymbol{\nu} & =\frac{1}{\sqrt{1+\tilde{u}_{x}^{2}}}\left(-\tilde{u}_{x}, 1\right), \\
\tilde{\kappa} & =\frac{\tilde{u}_{x x}}{\left(1+\tilde{u}_{x}^{2}\right)^{3 / 2}}
\end{aligned}
$$

and the parabolic equation

$$
\tilde{u}_{\tau}=\frac{\tilde{u}_{x x}}{1+\tilde{u}_{x}^{2}}-x \tilde{u}_{x}+\tilde{u}
$$

Fixed a small $\xi>0$, we consider the solution on the sub-interval $(-\ell+\xi$, $\ell-\xi)$. By concavity of the graph, we have

$$
\sup \left\{\left|\tilde{u}_{x}\right|: x \in(-\ell+\xi, \ell-\xi)\right\} \leq \frac{1}{\xi} \operatorname{osc}\{\tilde{u}(x, \tau): x \in(-\ell, \ell)\} \leq \frac{M}{\xi} .
$$

Hence, (3.6) is uniformly parabolic. By parabolic regularity theory, we know that all higher derivatives of $\tilde{u}$ are uniformly bounded in $(-\ell+\xi, \ell-\xi)$. The details are exactly as in the proof of Lemma 2.4. In particular, the curvature at the tip, when $x=0$, is uniformly bounded. By Propositions 2.2 and 2.3, we know that $\tilde{\gamma}$ converges to the $m$-circle, contradicting Lemma 2.2 and the maximum principle.

The contradiction shows that $\min _{\theta} \tilde{h}\left(\theta, \tau_{1}\right)=0$. Clearly, for all $\tau>\tau_{1}$, we have $\min _{\theta} \tilde{h}(\theta, \tau)<0$ and the evolution of the curve enters the third stage in figure $2 \mathrm{~b}$.

Next, we would like to make sure the solutions to problems (1.3) and (1.4) exist as long as the leaves do not shrink to points. Since the algebraic area may become negative, it is necessary to look at the unnormalized equation (1.3). 
Proposition 3.2. Let $h$ be a solution to the initial value problem (1.3). It exists as long as the area enclosed by a leaf does not vanish.

Proof. Suppose otherwise, then there is $t_{*}>0$ such that actual area of a leaf $\geq C>0$ and $\lim _{t \rightarrow t_{*}^{-}} \kappa(t)=\infty$. We represent the curve at time $t, \gamma(\cdot, t)$ as a concave graph as before. There is $\delta>0$ such that for $t \in\left(t_{*}-\delta, t_{*}\right)$ and $x \in(-\ell+\xi, \ell-\xi)$, we have the evolution equation

$$
u_{t}=\frac{u_{x x}}{\sqrt{1+u_{x}^{2}}},
$$

which is equivalent to (1.3); see, for example,[8]. Again, by similar argument as above, $u_{x}$ is uniformly bounded for $x \in(-\ell+\xi, \ell-\xi)$ and $t \in\left(t_{*}-\delta, t_{*}\right)$. Thus, we obtain a uniform bound for $\kappa(0, t)=\left.\frac{u_{x x}}{\sqrt{1+u_{x}^{2}}}\right|_{x=0}$. By continuity, as $t \rightarrow t_{*}^{-}, \kappa(0, t) \rightarrow$ a finite value, which is a contradiction.

Corollary 3.1. Let $t_{1}$ be corresponding to $\tau_{1}$ in Proposition 3.1. There is $t_{\infty}>t_{1}$ such that every leaf of the curve shrinks to a point.

Proof. Since $h\left(\theta, t_{1}\right)>0$ for all $m \pi / n \neq \theta \in[0,2 m \pi / n]$, we have $A\left(h\left(\cdot, t_{1}\right)\right)$ $>0$. By continuity, there is $\delta>0$ such that $A(h(\cdot, t))>0$ for $t<t_{1}+\delta$. Therefore, the area of a leaf of the curve at time $t<t_{1}+\delta$ is still positve. According to the above proposition, solution to (1.3) still exists for $t<t_{1}+\delta$. On the other hand, it is easy to see that the rate of change of the area of a leaf is less than $-\pi[1]$. Thus, there is a time $t_{\infty}>t_{1}$ that all the $m$ leaves disappear.

Remark . An affine version of the curve shortening flow is studied by Andrews [3], also de Lime and Montenegro,[14]. In the latter paper, a classification theorem parallel to the Abresch-Langer theorem for contracting self-similar curve along the affine flow is established. It is interesting to study whether the saddle point property still holds for these curves.

\section{Acknowledgment}

The discussions from our colleagues have been very encouraging and insightful in our study. We would like to specially mention K. S. Chou and Tom Wan. 


\section{References}

[1] U. Abresch and L. Langer, The normalized curve shortening flow and homothetic solutions, J. Differential Geom. 23(2) (1986), 175-196.

[2] S. Altschuler, Singularities of the curve shortening flow for space curves, J. Differential Geom. 34 (1991), 491-514.

[3] B. Andrews, The affine curve-lengthening flow, J. Reine AngEw. Math. 506 (1999), 43-83.

[4] S. Angenent, Parabolic equations for curves on surfaces I: Curves with p-integrable curvature. Ann. Math. 132(3) (1990), 451-483.

[5] S. Angenent, Parabolic equations for curves on surfaces II: Intersections, flow up and generlized solutions, Ann. Math. 133(1) (1991), $171-215$.

[6] S. Angenent, On the formation of singularities in the curve shrinking flow, J. Differential Geom. 33 (1991), 601-633.

[7] S. Angenent and J. Velázquez, Asymptotic shape of cusp singularities in curve shortening, Duke Math. J. 77(1) (1995), 71-110.

[8] Chou, K. S. and Zhu, X. P. The Curve Shortening Problem, Chapman \& Hall/CRC Press, Boca Raton, 2001.

[9] C. Epstein and M. Weinstein, A stable manifold theorem for the curve shortening equation, Comm. Pure Appl. Math. 40 (1987), 119-139.

[10] M. Gage and R. Hamilton, The heat equation shrinking convex plane curves, J. Differential Geom. 23 (1986), 69-96.

[11] M. Grayson, The heat equation shrinks embedded plane curves to round points, J. Differential Geom. 26 (1987), 285-314.

[12] N. V. Krylov and M. V. Safonov, Certain properties of solutions of parabolic equations with measurable coefficients, Izv. Akad. Nauk SSSR Ser. Mat. 44 (1) (1980), 161-175.

[13] G. M. Lieberman, Second Order Parabolic Differential Equations. World Scientific, Singapore, 1996.

[14] L. L. de Lima and J. F. Montenegro, Classification of solitons of the affine curvature flow, Comm. Anal. Geom. 7 (1999), 731-753. 
[15] L. Wang, On the regularity theory of fully nonlinear parabolic equations, Bull. Amer. Math. Soc. 22(1) (1990), 107-113.

Department of Mathematics

The Chinese University of Hong Kong

Shatin, Hong Kong.

E-mail address: thomasau@cuhk.edu.hk

ReCeived June 23, 2009 
